\title{
Silicon nanowire biosensor platform to electronically sense biomolecules in real patient samples
}

\author{
Sven Ingebrandt ${ }^{1,2}$, Miriam Schwartz ${ }^{2}$, Thanh Chien Nguyen ${ }^{2}$, Xuan Thang Vu $^{2}$, Achim Müller ${ }^{1,2}$, Duy \\ Phu Tran', Jannick Wilhelm², Jessica Ka-Yan Law ${ }^{2}$, Dipti Rani ${ }^{1}$, Vivek Pachauri ${ }^{1}$ \\ ${ }^{1}$ Department of Computer Sciences and Microsystem Technology, University of Applied Sciences \\ Kaiserslautern, 66482 Zweibrücken, Germany \\ ${ }^{2}$ RAM Group DE GmbH, Research and Development Center, 66482 Zweibrücken, Germany
} sven.ingebrandt@hs-kl.de

\begin{abstract}
:
In the past 10 years we developed a top down process on 4" wafer scale for the fabrication of silicon nanowire field-effect transistor arrays (SiNW FET). The devices are realized by etching techniques from silicon-on-insulator wafers. The SiNW FET structures and the contact lines are realized in one step using nanoimprint lithography and wet anisotropic etching of silicon. We optimized our process in several fabrication rounds in terms of process yield and reproducibility of the sensor structures. This enables differential readout between electrically identical devices. We typically utilize a combined potentiometric and impedimetric readout of the devices. In several test assays it became obvious that for reliable recordings in physiological buffer solutions also the detection and the control of side parameters such as $\mathrm{pH}$, temperature and ionic strength of the test solution are necessary. Our platform is now ready to enter into real patient sample tests.
\end{abstract}

Key words: Biosensor, silicon nanowires, label free detection, physiological buffer

\section{Introduction}

Silicon field-effect transistors with dimensions from micro- to nanoscale are widely used as transducers in the field of biosensors. Since the first introduction of ion-sensitive field-effect transistors (ISFETs) [1], they have been applied to many different applications. Very often the assays utilized enzymatic reactions such that secondary responses like surface-near $\mathrm{pH}$ changes were recorded. In general, the $\mathrm{pH}$ sensitivity is the primary response of ISFETs, since the typically-used gate oxides are sensitive to protonation and de-protonation in an electrolyte solution. The commonly used transducer principle for detection of biomolecules is potentiometry, where changes in surface potential at the liquid-solid interface are sensed and related to changes in sourcedrain currents of the devices. In affinity-based binding assays this method is preferentially used to detect small, charged biomolecules.

In the last decade various kinds of nanowire sensors were introduced - most of the time silicon nanowires. In many of the articles in this field, conductance changes of the wires upon biomolecule binding are displayed in a timedependent readout mode. In some publications remarkable sensitivities were reported.

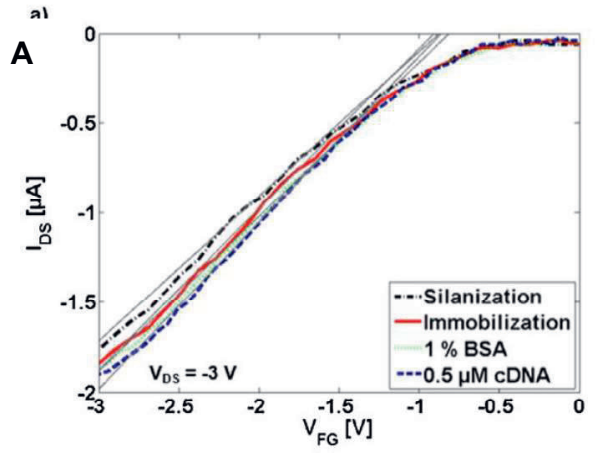

B

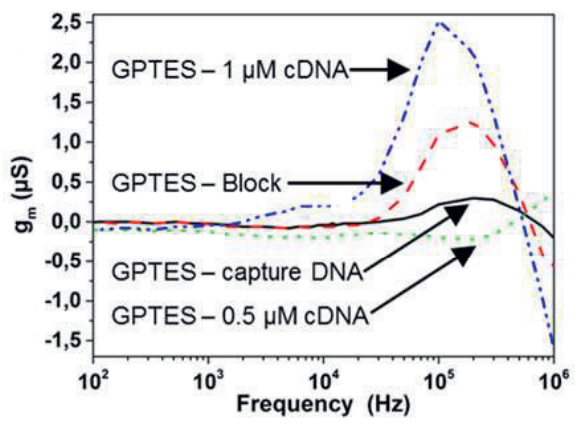

Fig. 1. Typical result for a DNA experiment using SiNW FET sensors. In a) a potentiometric readout is shown. The binding of DNA leads to a shift of the transfer characteristics of the SiNW FET (figure taken from [8]). In b) a typical experiment for impedance readout with SiNW FET devices upon DNA detection is shown. 
However, this standard readout principle is also highly prone to side effects originating from temperature, $\mathrm{pH}$ and ionic strength changes in the test liquid. It has also been discussed that some of the time-scales for the reported biomolecule detection assays in the given concentration regimes of analytes are at least questionable [2]. Indeed the transfer of this remarkable sensor concept towards real applications is yet to come - a timespan of more than 15 years hast past from the first publication [3].

\section{Results and Discussion}

In our project we aim to combine various sensor inputs to calibrate and compensate for the main important side effects and to work out the real concentration of analyte biomolecules. As a first step we combine potentiometric and impedimetric readout [4] for our SiNW FET platform (Fig. 1) [5-8]. The AC response of a field-effect transistor sensor is complicated to interpret, since not only the input impedance of the nanowire surface but also the parasitic contributions of reference electrode, electrolyte solution, contact line capacitance on chip, amplifier bandwidth and gain are playing a major role. A first approach to also model the contributions of these side effects we described in an earlier work. [9]

In a very recent work we increased the reliability and yield of our fabrication process and realized 32-channel SiNW FET devices (Fig. 2). [10]

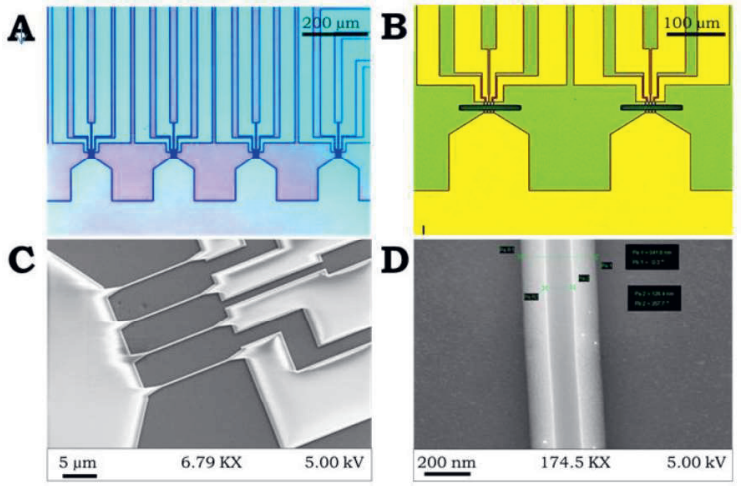

Fig. 2. 32-channel SiNW FET platform: The topdown fabricated SiNW FETs are all individually addressable. We used 8 groups of 4 channels separated by $250 \mu \mathrm{m}$ distance to enable microspotting of capture biomolecules.

At the moment we are aiming to combine the potentiometric and impedimetric readout principles in assays in a simultaneous readout mode. The rational is that the main interfering parameters to disturb the experiments for biomolecule detection are $\mathrm{pH}$ and temperature changes in the potentiometric mode while the ionic strength of the solution plays the major role in the impedimetric mode. Combining both readout methods in a multi-parametric readout approach might enable us to cancel out the influence of side effects and improve the reliability and robustness of the bioassays. In addition we aim for the integration of control sensors to our platform for temperature, $\mathrm{pH}$ and ionic strength to read out these effects and to stabilize the assay conditions. The necessary sensor structures are already realized and we currently work on the integration of the readout system.

The early works in the field of silicon nanowires also utilized a 'potentiometric' readout using certain $A C$ sensing principle at a fixed frequency [2]. It has been recently shown that in particular the impedimetric approach at elevated frequencies offers a sensing region, which is further extended into the electrolyte solution as compared to the potentiometric readout $[11,12]$. The reason for this effect is that in the impedimetric readout at higher frequencies the sensors do not only detect the charges of the molecules but the effect is based on an alternative physical principle known as dielectric spectroscopy. This method is detecting the change in dielectric constant close to the liquid-solid interface when biomolecules are binding and therefore it is not restricted by the typical Debye screening of charged like the potentiometric readout mode. First assays show that with this technique the detection of biomolecules in physiological solutions is possible.

\section{Conclusion and Outlook}

For precise readout of the biomolecular binding events with SiNW FET sensors, the methods of potentiometry and impedimetry can be combined and read out simultaneously. The latter one has been described to overcome the typical Debye screening effect of charges in an electrolyte solution by using an alternative transducer effect named dielectric spectroscopy. Both readout principles can be combined and a multivariant data analysis scheme can be applied to extract the biomolecule concentrations of a test assay. A complete sensor system should also detect and control the main important side parameters such as temperature, $\mathrm{pH}$ and ionic strength of the test solution. Biomolecules of different size such as DNA and proteins can be distinguished and detected by this novel approach.

\section{Acknowledgements}

We like to acknowledge financial support by the EUROIMMUN Medizinische Labordiagnostika AG, Germany, and by the European Commission Program through the Marie Curie 
Initial Training Network PROSENSE (grant no. $317420,2012-2016)$. Various other projects by University of Applied Sciences Kaiserslautern (UASK) and several BMBF projects funded the past research works of $\mathrm{PhD}$ students in our team. These persons in addition to the persons in the author list largely contributed to the development of the SiNW FET platform. In addition the technical staff of UASK is acknowledged for support.

\section{References}

[1] P. Bergveld, Development of an Ion-Sensitive Solid-State Device for Neurophysiological Measurements. IEEE Transactions on Biomedical Engineering 17, 70-71 (1970); doi: 10.1109/TBME.1970.4502688

[2] P. R. Nair, M. A. Alam, Performance limits of nanobiosensors, Applied Physics Letters 88, 233120 (2006); doi: 10.1063/1.2211310

[3] Y. Cui, Q. Wei, H. Park, C. M. Lieber, Nanowire nanosensors for highly sensitive and selective detection of biological and chemical species, Science 293, 1289-1292 (2001); doi: 10.1126/science.1062711

[4] S. Ingebrandt*, Y. Han, F. Nakamura, A. Poghossian, M. J. Schöning, A. Offenhäusser, Label-free detection of single nucleotide polymorphisms utilizing the differential transfer function of field-effect transistors, Biosensors and Bioelectronics 22, 2834-2840 (2007); doi: 10.1016/j.bios.2006.11.019

[5] X. T. Vu, J. F. Eschermann, R. Stockmann, R. GhoshMoulick, A. Offenhäusser, S. Ingebrandt, Top-down processed silicon nanowire transistor arrays for biosensing, Physica Status Solidi A 206, 426-434 (2009); doi: 10.1002/pssa.200880475.

[6] X.T. Vu, R. GhoshMoulick, J.F. Eschermann, R. Stockmann, A. Offenhäusser, S. Ingebrandt, Fabrication and application of silicon nanowire transistor arrays for biomolecular detection, Sensors and Actuators B: Chemical 144, 354-360 (2010); doi:10.1016/j.snb.2008.11.048

[7] X. T. Vu, R. Stockmann, B. Wolfrum, A. Offenhäusser, S. Ingebrandt, Fabrication and application of a microfluidic-embedded silicon nanowire biosensor chip, Physica Status Solidi A 207, 850-857 (2010); doi: 10.1002/ pssa.200983316

[8] M. Schwartz, T. C. Nguyen, X. T. Vu, M. Weil, J. Wilhelm, P. Wagner, R. Thoelen, S. Ingebrandt*, DNA detection based on a potentiometric readout method with silicon nanowire arrays in linear operation regime, Physica Status Solidi A 213, 1510-1519 (2016); doi: 10.1002/pssa.201532919

[9] T. C. Nguyen, X. T. Vu, M. Freyler, S. Ingebrandt, PSPICE model for silicon nanowire based biosensors in impedimetric measurement mode, Physica Status Solidi A 210, 870-876 (2013); doi: 10.1002/pssa.201200919
[10] D. Rani, V. Pachauri, A. Mueller, X. T. Vu, S. Ingebrandt, On the use of scalable NanoISFET arrays of silicon with highly reproducible sensor performance for assay-based applications, ACS Omega 1, 244-250 (2016); doi: 10.1021/acsomega.6b00014

[11] C. Laborde, F. Pittino, H. A. Verhoeven, S. G. Lemay, L. Selmi, M. A. Jongsma, F. P. Widdershoven, Real-time imaging of microparticles and living cells with CMOS nanocapacitor arrays, Nature Nanotechnology 10, 791-795 (2015); doi:10.1038/nnano.2015.163

[12] S. Ingebrandt, Bioelectronics: Sensing beyond the limit, Nature Nanotechology 10, 734 (2015); doi:10.1038/nnano.2015.199 